Case

\title{
Sinus Node Artery-preserving Superior Transseptal Approach: A Simple Technique
}

\author{
Ryusuke Suzuki, MD, Toshiaki Watanabe, MD, Mai Matsukawa, MD, \\ Keiko Hiroshige, MD, Sojiro Sata, MD, and Toshiya Koyanagi, MD
}

\begin{abstract}
With the use of the superior transseptal approach during mitral valve surgery, good exposure of the mitral valve can be achieved with simple traction sutures, which minimize the risk of deformation of the mitral valve. For this reason, we routinely perform mitral valvoplasty using the superior transseptal approach; however, we, occasionally encounter cases that develop postoperative atrial dysrhythmia. We have therefore, devised a very simple technique for preservation of the sinus node artery in the superior transseptal approach, which is effective for reducing the incidence of postoperative sinus node dysfunction. In this technique, during incision of the dome of the left atrium, the sinus node artery is carefully dissected and preserved.
\end{abstract}

Keywords: mitral valve, arrhythmia, sinus node artery

\section{Introduction}

Surgical success in cardiovascular surgery is related to the obtainment of a good surgical field, particularly in the case of surgery on the mitral valve, because anatomically, the left atrium is located in the posterior region of the heart. In addition, visualization of the mitral valve in a small atrium or during reoperation can be of critical importance.

Several approaches have been used for surgery in this area, including the transseptal approach, right-side/leftatrium approach and superior transseptal approach, and numerous opinions have been expressed on their respective advantages in a mitral valve operation; in particular, the superior transseptal approach provides the best field

\footnotetext{
Department of Cardiovascular Surgery, Japanese Red Cross Kumamoto Hospital, Kumamoto, Kumamoto, Japan
}

Received: April 14 2010; Accepted: August 20, 2010

Corresponding author: Ryusuke Suzuki, MD. Department of Cardiovascular Surgery, Japanese Red Cross Kumamoto Hospital, 2-1-1 Nagamine-Minami, Kumamoto, Kumamoto 861-8520, Japan Email: rskszk@hotmail.com

(C)2011 The Editorial Committee of Annals of Thoracic and Cardiovascular Surgery. All rights reserved. of view for mitral valve surgery. However, division of the sinus node artery in this approach may be associated with sinus node dysfunction and necessitates pacemaker implantation in some patients. ${ }^{1,2)}$

In recent years, there have been an increasing number of cases of mitral valve repair for mitral valve regurgitation, for which excellent exposure of the mitral valve is essential. Through the superior transseptal approach, the mitral valve is exposed with simple traction sutures, minimizing the likelihood of deformation of the mitral valve. ${ }^{3)}$ For this reason, we routinely perform mitral valvoplasty using the superior transseptal approach; however, we have occasionally encountered patients who exhibit postoperative atrial dysrhythmia.

In principle, we perform preoperative coronary angiography to examine the course of the sinus node artery. We have established a new technique for preservation of the sinus node artery while using the superior transseptal approach, and demonstrated the effectiveness of this technique in reducing the incidence of postoperative sinus node dysfunction.

\section{Technique}

The heart is exposed by a median sternotomy. Arterial 


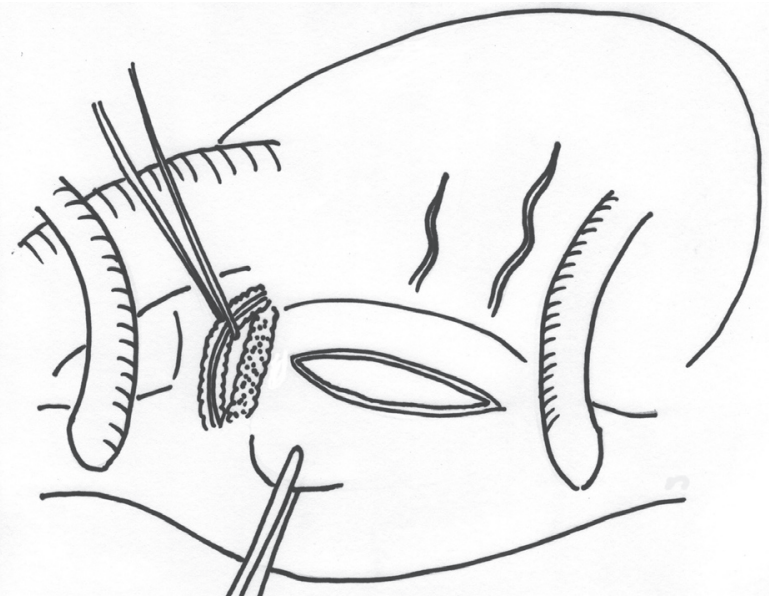

Fig. 1 The incision was carefully extended just up to the sinus node artery, and the sinus node artery was preserved with elastic vessel tape and dissected from the right atrial wall about $1 \mathrm{~cm}$ proximally and distally with a few surrounding tissue.

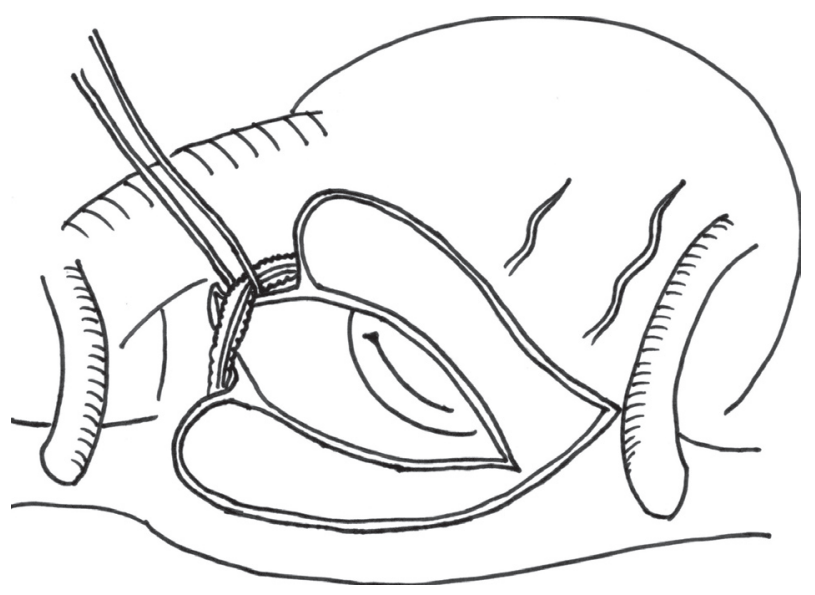

Fig. 2 The mitral valve was exposed as easily as in the normal transseptal superior approach.

cannulation of the ascending aorta is performed, and selective venous cannulation is accomplished via the superior vena cava and through the right atrium near the inferior vena cava. Snares are placed around both the inferior and superior vena cava. A venting tube is placed via the right superior pulmonary vein. Antegrade cardioplegia is used in all patients. The right atriotomy is performed parallel to the atrioventricular groove and the incision is carried superiorly, medial to the right atrial appendage. After the incision is carefully extended to the sinus node artery, the sinus node artery is preserved with elastic vessel tape and dissected from the right atrial wall

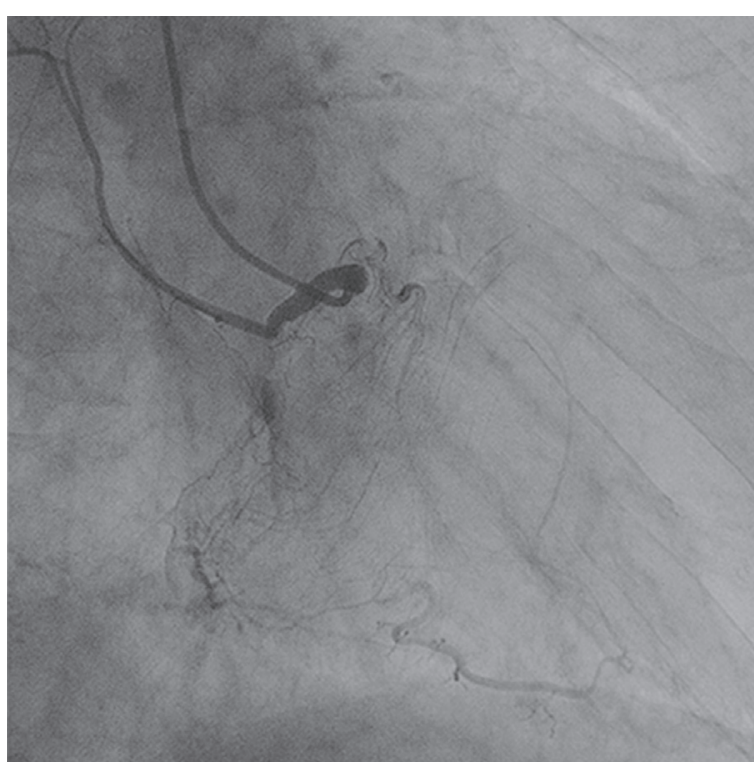

Fig. 3 Case 1: Preoperative coronary angiography. The SNA arises from the right coronary artery. SNA: sinus node artery

about $1 \mathrm{~cm}$ proximally and distally with a few surrounding tissue. The left atrial incision is begun in the fassa ovalis of the atrial septum and extended vertically and superiorly where it joins the right atrial incision (Fig. 1). The left atrial incision is extended into the superior dome of the left atrium underneath the ascending aorta (Fig. 2). In general practice, the mitral valve is exposed using a few traction sutures (4-0 polypropylene), as easily as in the normal transseptal superior approach. The right side wall traction is accomplished carefully so as not to expand the sinus node artery. After completion of the mitral valve procedure, the left atrium is closed with a 4-0 polupropylene suture using caution so as not to injure the sinus node artery, and sometimes, a reinforcing suture is also placed, because the right atrial wall is relatively thin.

\section{Case}

A 66-year-old man who had aortic and mitral valve regurgitation was scheduled to undergo aortic valve replacement and mitral valvoplasty. Preoperative coronary angiography showed that the sinus node artery arose from the right coronary artery (Fig. 3). During the operation, we identified the sinus node artery on the wall of the right atrium (Fig. 4). The mitral valve was exposed using the superior transseptal approach. When the cranial wall of the left atrium was dissected, the sinus node artery was carefully separated, and traction was exerted on it using 


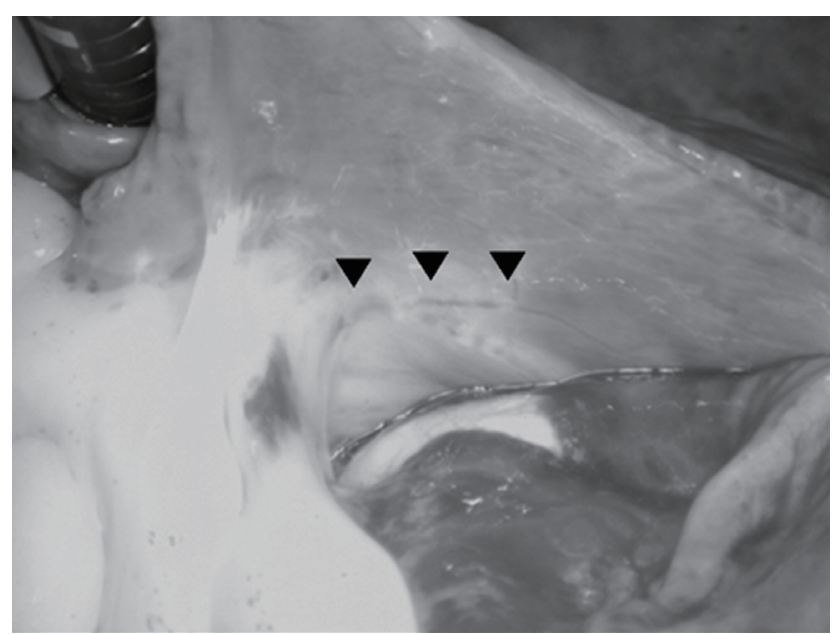

Fig. 4 Case 1: Intraoperative view.

The SNA ( $\Delta$ ) can be detected easily on the cranial wall of the right atrium.

SNA: sinus node artery

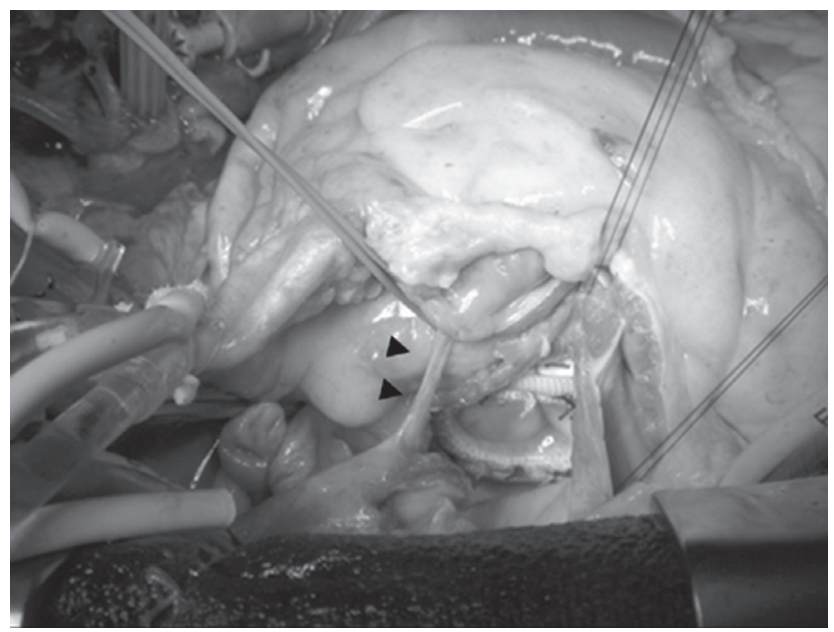

Fig. 5 Case 2.

Using the transseptal superior approach, mitral valve replacement could be easily achieved without cutting the SNA ( $(\boldsymbol{\Delta})$.

SNA: sinus node artery

vessel tape. The patient exhibited normal sinus rhythm preoperatively, which was subsequently maintained.

\section{Comment}

Good exposure of the mitral valve and avoidance of postoperative rhythm disturbance are both very important for cardiac surgeons. A large number of papers describing the anatomical structure of the sinus node artery have

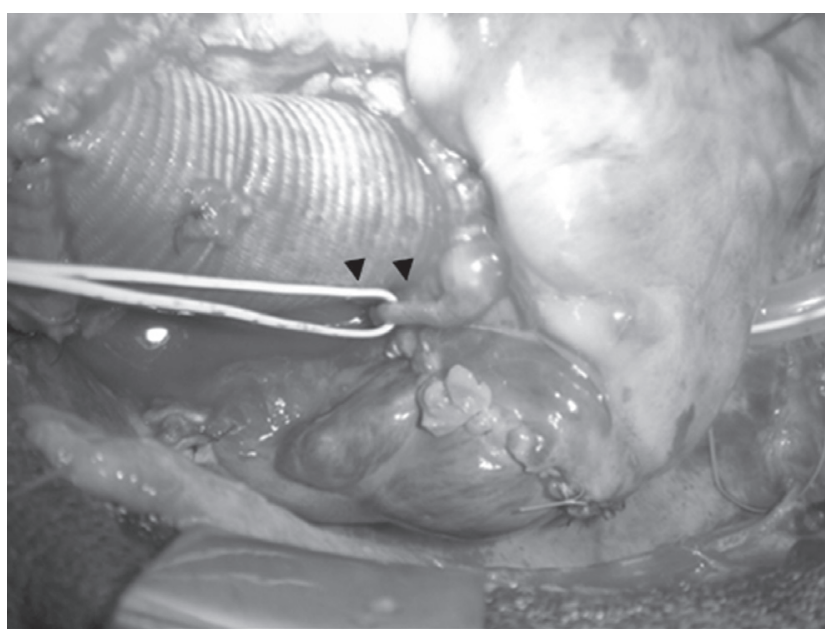

Fig 6 Case 3

SNA ( $\boldsymbol{\Delta})$-preserving transseptal superior approach with concomitant modified Bentall procedure. The atriotomy could be sutured without injury to the SNA.

SNA: sinus node artery

been published. Berdajs et al. ${ }^{4}$ classified the origins of the sinus node artery and reported that the majority arose from the right coronary artery and ran along the anterior wall of the right atrium toward the orifice of the superior vena cava; indeed, coronary angiography often defines this course. Therefore, the sinus node artery can be cut during the use of the usual superior transseptal approach. Kumar et al. ${ }^{5)}$ reported that the occurrence of transient junctional rhythm in the early postoperative period is not rare. The relationships between sinus node function, atrial arrhythmias, and various surgical approaches for exposure of the mitral valve have been examined critically.

Preoperative coronary angiography to determine whether the sinus node artery passes along the anterior wall of the right atrium is very important for preservation of the sinus node artery. We generally pay attention to avoiding deep transection of the superior wall of the left atrium, because the accessory branch runs along the left side of the superior vena cava. In this operation, we were able to identify the sinus node artery on the wall of the right atrium and preserved it. Preservation of the sinus node artery is a prerequisite for avoiding sinus node dysfunction; however, the question of the conduction system remains an unresolved problem. Additional case reports of sinus node function in patients undergoing mitral valve surgery through the superior transseptal approach are required. We have already operated on three patients using this technique, and none of these patients exhibited sinus node dysfunction postoperatively (Fig. 5 and $\mathbf{6}$ ). 
However, prior to this case series, we encountered one case in which the sinus node artery was unfortunately divided as it ran through the muscle layer of the right atrium. That patient was implanted with a pacemaker to control the sinus node dysfunction. We consider that difficulties are more likely to occur when the sinus node artery runs through the muscle layer of the right atrium.

\section{References}

1) Lukac P, Hjortdal VE, Pedersen AK, et al. Superior transseptal approach to mitral valve is associated with a higher need for pacemaker implantation than the left atrial approach. Ann Thorac Surg 2007; 83: 77-82.

2) Takeshita M, Furuse A, Kotsuka Y, et al. Sinus node function after mitral valve surgery via the transseptal superior approach. Eur J Cardiothorac Surg 1997; 12: 341-4.

3) Kon ND, Tucker WY, Mills SA, et al. Mitral valve operation via an extended transseptal approach. Ann Thorac Surg 1993; 55: 1413-7.

4) Berdajs D, Patonay L, Turina MI. The clinical anatomy of the sinus node artery. Ann Thorac Surg 2003; 76: 732-6.

5) Kumar N, Saad E, Prabhaker G, et al. Extended transseptal versus conventional left atriotomy: early postoperative study. Ann Thorac Surg 1995; 60: 426-30. 